\title{
Current theories on the cause of Parkinson's disease
}

\author{
J WILLIAM LANGSTON \\ From the California Parkinson's Foundation, San Jose, CA, USA
}

In this article, I will attempt the somewhat daunting task of delineating current theories on the sequence of events that lead to Parkinson's disease. Any review of this nature must border on a discussion of the cause of the disease which, of course, remains unknown. Nonetheless, with our increasing knowledge about the nigrostriatal system, both in normality and disease, it seems that the time is right to put some of the pieces together in an attempt to narrow down the factors that might eventually lead to what we know as Parkinson's disease.

\section{Aging}

One cannot discuss the cause of Parkinson's disease without also taking into consideration the importance of aging. This is true for two reasons. First, Parkinson's disease is primarily a disease of older individuals; it is rare before the age of 40 years, and the incidence rate increases at least until the sixth or seventh decade of life. Thus, aging itself appears to be a key susceptibility factor for Parkinson's disease. Secondly, the major substrate upon which the disease appears to act, the dopaminergic nigrostriatal system, is itself affected by the aging process. Carlsson and Winblad' were the first to point out that there appears to be a gradual decline in striatal dopamine concentrations throughout life, progressing at a rate of approximately 5 to $8 \%$ per decade. Thus, within the Parkinsonian nigrostriatal system, there is an unavoidable mingling of the decline that occurs normally with aging, and loss of function that occurs as part of the disease process due to Parkinson's disease. This brings us to the first in a series of dilemmas that are encountered when attempting to sort out which factors are important in Parkinson's disease and which are not.

First, the reason for the normally-occurring agerelated decline in striatal dopamine, which may or may not reflect a loss of dopaminergic neurons in the substantia nigra, is not known. One possibility is that

Address for reprint requests: Dr J William Langston, California Parkinson's Foundation, 2444 Moorpark Avenue, Suite 316, San Jose, California 95128, USA.

Accepted February 1989 this process is genetically programmed. If this were the case, it would represent a true aging phenomenon, as aging itself is probably under genetic control. Interestingly, the catecholaminergic nuclei of the brainstem were at one time suggested as possible "pacemakers"2 for the aging process. If the process is indeed preprogrammed, then it would be of great interest to know exactly how the decline is orchestrated.

A second possibility is that this system, through some intrinsic defect, is particularly prone to injury. The two most commonly cited factors that might lead to excessive wear and tear on the nigrostriatal dopaminergic system are free radical production and the accumulation of neuromelanin. The free radical hypothesis, of course, has its origin in the fact that striatal dopamine concentrations are particularly high compared with other areas of brain; for each mole of dopamine that is oxidised by monoamine oxidase (MAO), a mole of hydrogen peroxide is generated. This excess of hydrogen peroxide, and possibly other oxyradicals, could be damaging to neurons. Although there are a number of enzymes that protect against free radicals, including superoxide dismutase and glutathione peroxidase, it does seem possible that with time these systems might fail or at least prove inadequate to completely prevent long-term damage from free radical formation. Auto-oxidation of dopamine also results in the formation of quinones, which themselves may be toxic, ${ }^{3}$ probably through a mechanism of covalent binding. Thus, there are several potentially damaging mechanisms which might eventually take their toll on nigrostriatal neurons during life. This point was made some ten years ago by Doyle Graham, ${ }^{4}$ who pointed out that degeneration of nigrostriatal neurons might occur as a result of lifelong injury from these by-products of dopamine oxidation. However, since everyone is exposed to this life-long process of catecholamine oxidation, this hypothesis might be better equipped to explain the neuronal decline that occurs normally throughout the life of the nigrostriatal dopaminergic system, rather than that which occurs in those individuals affected by Parkinson's disease.

Neuromelanin represents another factor that could compromise neuronal function over time. This complex substance, which is thought to be comprised of 
dopamine polymers and lipofuscin, accumulates at least through the sixth to seventh decades of life in the substantia nigra and locus ceruleus. ${ }^{5}$ While neuromelanin has been implicated in Parkinson's disease, because this substance accumulates as a normal phenomenon, its deleterious effects (if any) may also be better suited to explain the decline in nigrostriatal function that occurs with normal aging. On the other hand, it does seem possible that neuromelanin may make neurons particularly vulnerable to the agent(s) that cause Parkinson's disease. For example, Hirsch et $\mathrm{al}^{6}$ have recently shown that there appears to be an almost direct relationship between the distribution of neuromelanin-bearing cells in the substantia nigra, and the pattern of cell loss in Parkinson's disease.

One conclusion that can be reached from the foregoing is that both the free radical hypothesis and neuromelanin hypothesis are most easily invoked to explain the normal aging phenomenon rather than Parkinson's disease itself. If either or both of these factors are at play in Parkinson's disease, there must be a reason why their detrimental effects accelerate in certain individuals, eventually causing Parkinson's disease. An inherited defect that bestows exceptional vulnerability to either or both of these factors seems unlikely in view of recent twin studies that failed to show any evidence of genetic transmission of Parkinson's disease.? Thus, if any of the factors that cause the normal age-related decline in striatal dopamine are at play in the idiopathic disease, we are led to the inescapable conclusion that a trigger factor capable of causing an acceleration of the normal aging phenomenon is involved. Mann and Yates ${ }^{8}$ recently reached a similar conclusion, suggesting that there must be secondary pathogens to exacerbate the effects induced by the accumulation of neuromelanin, if this substance indeed plays a causative role in Parkinson's disease.

Finally, it is not yet certain that the substrate of a normally declining nigrostriatal system is even necessary for Parkinson's disease to develop. However, since the issue of what causes normal age-related decline appears to be such an important one (indeed, it may be impossible to sort out what is due to Parkinson's disease and what is normal aging without knowing the cause of the aging phenomenon), perhaps research efforts should focus more on the cause of the normal age-related decline, thus allowing us to put an essential piece of the puzzle in place before we pursue our studies of Parkinson's disease further. Only then might we be able to address the key question of whether or not the process responsible for nigrostriatal degeneration during aging is the same or different from that which causes Parkinson's disease. It should also be noted that, while this discussion is focused primarily on dopamine depletion in the striatum, it is still unclear if the decline in striatal dopamine reflects a loss of nigral neurons, or simply a decline in cellular function; this important question also warrants further study.

\section{Aging plus an environmental insult}

An alternative explanation to an acceleration of an age-related decline in the nigrostriatal system is the hypothesis put forth by Calne and Langston ${ }^{9}$ several years ago. This hypothesis proposes that the substantia nigra is damaged as the result of an environmental insult at some point early in life, resulting in a partial loss of dopaminergic neurons; the insult would remain asymptomatic unless it was adequate to produce greater than the $80 \%$ depletion of striatal dopamine required to induce clinical evidence of Parkinsonism. In this situation, if the normal agerelated decline in striatal dopamine continued unabated, the $80 \%$ threshold might be reached many years later.

This hypothesis predicts that the rate of cell loss in Parkinson's disease and in normal aging should be the same; indeed, one could argue it might be even less in Parkinson's disease, as there are fewer cells remaining to undergo degeneration. While gliosis itself is not a good monitor of a rate of disease progression, one can refine this question by studying microglia. A proliferation of microglia generally indicates an active process. This is particularly true if one looks for microglial nodules, which usually indicate neuronophagia. Recently, McGeer and colleagues ${ }^{10}$ have studied this question using quantitative techniques. By staining microglia using immunohistochemical techniques for HLA-DR, these investigators showed that a number of positive cells in Parkinsonian brains were $196 \pm 66$ per 5 high-power fields, as opposed to $16 \pm 12$ in agematched controls. If the counts were limited to neuronophagias, the numbers were still at least six times higher in patients with Parkinson's disease. Thus, it appeared that the rate of neuronal cell degeneration was considerably higher in Parkinsonian patients than could be accounted for on the basis of normal age-related neuronal degeneration alone. These results argue against the hypothesis that normal aging alone can account for the appearance of Parkinson's disease many years after a partial insult to the substantia nigra.

However, there is an elaboration of this hypothesis that resolves this problem. After partial cell loss of nigral neurons, one might expect increased dopamine turnover in the remaining neurons as a compensatory mechanism. This, of course, would result in increased oxidative stress which, in turn, might lead to additional cell loss. Thus, a vicious cycle could be set in 으음 읃 ㅁำ 过 
motion, accelerating the process of neuronal loss. Thus, after an environmental insult, rather than an age-related decline that parallels that which is seen with normal aging, there could be an acceleration of this process, thereby explaining the data of McGeer et al. ${ }^{10}$

Hypotheses are not very useful if they cannot be tested. For this reason, the DATATOP study ${ }^{11}$ which involves the testing of both deprenyl and alpha-tocopherol in early, untreated Parkinson's disease, is particularly important. This double-blind, placebocontrolled trial should allow us to determine for the first time whether therapeutic intervention with a free radical scavenger (in this case alpha-tocopherol) might actually retard the process of neuronal degeneration in Parkinson's disease. A positive result would support the hypothesis that free radical generation may be providing one of the driving forces behind nigral cell degeneration in the disease. On the other hand, a negative result will be less useful, as one could argue that the wrong antioxidant was chosen or the doses were inadequate. In any case, there is a reasonable chance that this hypothesis may be adequately tested and answers could be forthcoming within the next few years.

\section{MPTP}

Laboratory research with the neurotoxin MPTP (1methyl-4-phenyl-1,2,3,6-tetrahydropyridine) has suggested a different explanation that might explain the sequence of events that lead to Parkinson's disease. It was first observed in 1985 that the effects of MPTP are more pronounced when the compound is given to older animals. ${ }^{12}{ }^{13}$ For example, in our studies, we saw little evidence of neuronal degeneration in young mice given MPTP, but pronounced evidence of cell death in older animals. ${ }^{14}$ This age dependency has been reported by other investigators as well, ${ }^{15}$ and raises the possibility of an entirely different sequence of events leading to Parkinson's disease. If the disease is indeed due to an environmental toxin, one could propose that constant exposure early in life would induce little in the way of neuronal degeneration. However, once the nervous system became increasingly susceptible to such a toxin by virtue of the aging process, its neurodegenerative effects might become manifest. This hypothesis still does not explain, however, why some individuals get the disease and others do not, unless it is simply a matter of who gets exposed to the causative agent and who does not. The issue remains a critical one in regard to the environmental hypothesis; for the answer, we may have to turn to our colleagues in the field of epidemiology.

MPTP has also given us cause to consider another phenomenon. It is now well known that MPTP is probably a protoxin, requiring conversion by MAO B to MPP +, the putative toxic metabolite of this compound. While most substances in the brain decline with normal aging, MAO B (but not A) has been shown, both in rodents ${ }^{16}$ and primates, ${ }^{17}$ including the human, ${ }^{18}$ to increase with aging. The mechanism of this increase is not understood, but it could provide a means whereby increasing amounts of a neurotoxin are generated from environmental exposure to a protoxin. Once again, the DATATOP study may provide help in answering this question, since the other drug to be tested is the selective MAO B inhibitor deprenyl. As a result of the DATATOP study, as well as a similar study that we are in the process of concluding, ${ }^{19}$ we should soon learn if blocking MAO B on a chronic basis will favourably alter the course of Parkinson's disease.

How chronic exposure to an MPTP-like toxin might occur is unclear, but one interesting possibility relates to the adrenal gland. In our studies in primates given MPTP, the adrenal gland contained approximately ten-fold as much MPP + as in any area of the body or brain; further, MPP + persists in the gland for a prolonged period of time. Similar results have been obtained by Johannessen and colleagues. ${ }^{20}$ It has now been shown that MPP + is stored in the chromaffin granules within the adrenal medulla. ${ }^{21}$ Is it possible that the adrenal gland might act as a sequestering pocket? If so, it could provide a source for the slow release of neurotoxin into the body and, perhaps, eventually the brain. As the list of toxic MPTP analogues continues to grow, it seems reasonable to suggest that this family of catecholaminergic toxins warrants close study. It will, of course, be of great interest to see if one or more of them are ever discovered to exist either in the man-made or natural environment.

\section{Lessons from Guam}

Currently, there is great interest in the aetiology of the amyotrophic lateral sclerosis-Parkinsonism-dementia (ALS/PD) complex of Guam..$^{22}$ In recent years there appears to be a declining incidence of this disease, suggesting the possibility that there is decreasing exposure to an environmental agent that causes the disease. Recently, Spencer and colleagues have presented evidence that BMAA, an excitatory amino acid found in the Cycad plant, which was previously used as a food source on Guam, induces some of the neuropathological features of the ALS/PD complex in primates after chronic exposure. ${ }^{23}$ While these studies are preliminary, they raise the interesting question whether or not an ingested toxin could be responsible for this progressive neurodegenerative disease.

These studies alone are certainly of interest, but 
their greatest relevance to Parkinson's disease may lie in the concept of long-latency neurotoxins. This concept emerged from the observation that individuals have developed ALS/PD for periods ranging up to 30 or more years after leaving the island of Guam. The implication is that a neurotoxin could conceivably exert its effects many years after exposure has occurred. It must be stressed that this is a concept which has yet to be proven, as it is not yet clear that BMAA, or any other neurotoxin, causes the ALS/PD complex. However, if the concept can be validated, it could have major implications for the evolution of events leading to Parkinson's disease. We may also have the opportunity to validate this concept by following a large cohort of individuals exposed to MPTP who currently have little or no evidence of Parkinsonism. ${ }^{24}$

The greatest challenge regarding the concept of long-latency neurotoxins is the exact mechanism by which such a process might occur. For catecholaminergic systems, one could have invoked the free radical hypothesis already discussed, but for other neuronal subpopulations, such as anterior horn cells in the spinal cord, a similar mechanism is not readily apparent. Nonetheless, validating the concept of long-latency neurotoxins, which has recently been challenged, ${ }^{25}$ is imperative if we are to consider seriously neurotoxicants as potential aetiologic agents in neurodegenerative diseases.

\section{Altering the genetic machinery of cells}

Should DATATOP fail to support the free radical hypothesis as a cause for progressive cell death in Parkinson's disease, we may have to look elsewhere for the mechanism whereby cells could slowly degenerate after an environment insult, whether this insult results from exposure to a neurotoxicant, or some other exogenous agent or event. One novel hypothesis that may deserve further investigation is that an environmental insult might alter the cell's ability to express its own genetic code. It seems unlikely that Parkinson's disease is due to a defect in DNA, as it is difficult to envisage how DNA would be selectively affected in the nigrostriatal neurons and nowhere else in the body. A cell with its full complement of DNA should be able to repair itself after a limited injury, as it would be capable of reconstituting deficient enzymes necessary for repair or replacement. However, recent evidence is emerging from studies of another neurotoxicant, alcohol, that it is possible to alter cellular genetic machinery in such a way that the cell is no longer able to translate its genetic code adequately, an event that could eventually interfere with cellular functioning, and perhaps someday result in cell death. In a sense, the cell could "never go home again" in terms of regulating its own genetic expression.

The data that support this hypothesis are the result of work with agonist-sensitive adenylate cyclase. It has been known for some time that this system can be down-regulated by chronic exposure to alcohol. Recently, it has been shown by Tabakoff and colleagues, ${ }^{26}$ using human platelets, that this downregulation can persist for a number of years after alcohol exposure (it is not yet absolutely certain that this down-regulation preceded the alcoholism, however). More recently, evidence has been reported indicating that this alteration is secondary to downregulation of messenger RNA synthesis. ${ }^{27}$ The important concept here is that it is apparently possible to alter selectively the cell's ability to produce messenger RNA, and that this alteration can last for years.

Applying this concept to Parkinson's disease, one could postulate that exposure to a neurotoxicant might permanently alter the neuron's own genetic machinery. This in turn might result in the inability to synthesise a critical enzyme in adequate amounts, eventually leading to the cell's demise. Of course these ideas are only speculative, but they may represent a future area of research, should our current efforts to explore the evolution of events leading to Parkinson's disease fail to bear fruit.

The author wishes to acknowledge gratefully the help of Pamela Schmidt and David Rosner in the preparation of the manuscript. This work is supported in part 8 by the Parkinson's Disease Foundation, the United Parkinson Foundation, the National Institute of Aging (RO1 AGO7348-01) and the National Institute $\rightleftharpoons$ for Environmental Health Sciences (RO1 ESO3697$03)$.

\section{References}

1 Carlsson A, Winblad B. Influence of age and time interval between death and autopsy on dopamine and 3-methyoxytyramine levels in human basal ganglia. $J$ Neurol Transm 1976;38:271-76.

2 Marx J. Aging research (II): Pacemakers for aging? Science 1974;186:1196-97.

3 Graham DG, Tiffany SM, Vogel FS. The toxicity of melanin precursors. J Invest Dermatol 1978a;70:113-16.

4 Graham DG, Tiffany SM, Bell WR Jr, Gutknecht WF. Autoxidation versus covalent binding of quinones as the mechanism of toxicity of dopamine, 6-hydroxydopamine, and related compounds toward $\mathrm{Cl} 300$ neuroblastoma cells in vitro. $\mathrm{Mol}$ Pharmacol 1978b;14:644-53.

5 Graham DG. On the origin and significance of neuromelanin. Arch Pathol Lab Med 1979;103:359-62.

6 Hirsch E, Graybiel AM, Agid YA. Melanized dopaminergic neurons are differentially susceptible to degeneration in Parkinson's disease. Nature 1988;334:345-48.

7 Ward CD, Duvoisin RC, Ince SE, Nutt JD, Eldridge R, Calne DB. Parkinson's disease in 65 pairs of twins and in a set of quadruplets. Neurology 1983;33:815-24.

8 Mann DMA, Yates PO. Possible role of neuromelanin in the pathogenesis of Parkinson's disease. Mech Aging Dev 1983; 
21:193-203.

9 Calne DB, Langston JW. Aetiology of Parkinson's disease. Lancet 1983;2:1457-59.

10 McGeer PL, Itagaki S, Akiyama H, McGeer EG. Rate of cell death in parkinsonism indicates active neuropathological process. Ann Neurol 1988;24:574-6.

11 Shoulson I. Experimental therapeutics directed at the pathogenesis of PD. In: Calne DB, ed. Handbook of Experimental Pharmacology: Drugs for the Treatment of PD. New York: Springer-Verlag, (in press).

12 Jarvis MF, Wagner GC. Age-dependent effects of 1-methyl-4phenyl-1,2,3,6-tetrahydropyridine (MPTP). Neuropharmacol 1985;24:581-83.

13 Ricaurte GA, Langston JW, Irwin I, DeLanney LE, Forno LS. The neurotoxic effect of MPTP on the dopaminergic cells of the substantia nigra in mice is age-related. Soc Neurosci Abstract 1985;11:631.

14 Ricaurte GA, Langston JW, DeLanney LE, Irwin I, Peroutka SJ, Forno LS. Fate of nigrostriatal neurons in young mice given 1methyl-4-phenyl-1,2,3,6-tetrahydropyridine (MPTP): A neurochemical and morphological reassessment. Brain Res 1986;376:117-24.

15 Gupta M, Gupta BK, Thomas R, Bruemmer V, Sladek JR Jr, Felten DL. Aged mice are more sensitive to 1-methyl-4-phenyl1,2,3,6-tetrahydropyridine treatment than young adults. Neurosci Lett 1986;70:326-31.

16 Strolin-Benedetti MS, Keane PE. Differential changes in monoamine oxidase $A$ and $B$ activity in the aging rat brain. $J$ Neurochem 1980;35:1026-32.

17 Cote LJ, Kremzner LT. Biochemical changes in normal aging in human brain. In: Mayeux R, Rosen WG, eds. The Dementias. New York: Raven Press, 1983:19-30.

18 Robinson DS, Nies A, Davis JN, Bunney WE, Davis JN, Colburn RW, Bourne HR, Shaw DM. Ageing, monoamines, and monoamine-oxidase levels. Lancet 1972;1:290-91.

19 Tetrud JW, Langston JW. L-deprenyl as a possible protective agent in Parkinson's disease. J Neural Transm 1987; Suppl 25: $1-20$.

20 Johannessen JN, Chiueh CC, Herkenham MA, Markey SP, Burns RS, Adams JD, Schuller HM. Relationship of the in vivo metabolism of MPTP to toxicity. In: Markey SP, Castagnoli Jr N, Trevor AJ, Kopin IJ, eds. MPTP: A neurotoxin producing a parkinsonian syndrome. New York: Academic Press, 1986: 173-89.

21 Reinhardt JF Jr, Diliberto Jr EJ, Viveros OH, Daniels AJ. Subcellular compartmentalisation of 1-methyl-4-phenylpyridinium with catecholamines in adrenal medullary chromaffin vesicles may explain the lack of toxicity to adrenal chromaffin cells. Proc Natl Acad Sci 1987;84:8160-4.

22 Lewin R. Environmental hypothesis for brain diseases strengthened by new data. Science 1987;237:483-4.

23 Spencer PS, Nunn PB, Hugon J, Ludolph AC, Ross SM, Dwijendra NR, Robertson RC. Guam amyotrophic lateral sclerosis-parkinsonism-dementia linked to a plant excitant neurotoxin. Science 1987;237:517-22.

24 Ruttenber AJ, Garbe PL, Kalter HD, Castro KG, Tetrud JW, Porter P, Irwin I, Langston JW. Meperidine analog exposure in California narcotics abusers: Initial epidemiologic findings. In: Markey SP, Castagnoli Jr, N, Trevor AJ, Kpin IJ, eds. MPTP: A neurotoxin producing a parkinsonian syndrome. New York: Academic Press, 1986:339-53.

25 Duncan MW, Kopin IJ, Garruto RM, Lavine L, Markey SP. 2Amino-3(methylamino)-propionic acid in cycad-derived foods is an unlikely cause of amyotrophic lateral sclerosis/parkinsonism. Lancet, September 10, 1988:631-2.

26 Tabakoff B, Hoffman PL, Lee JM, Saito T, Willard B, De Leon-Jones F. Differences in platelet enzyme activity between alcoholics and non-alcoholics. $N$ Engl $J$ Med 1988;318:134-9.

27 Mochly-Rosen D, Fu-Hsiung C, Cheevers L, Kim M, Diamond I, Gordon AS. Chronic ethanol causes heterologous desensitisation of receptors by reducing $\alpha_{s}$ messenger RNA. Nature 1988;333(6176):848-50. 